\section{Research Square}

\title{
Active Galactic Nuclei as Factories for Eccentric Black Hole Mergers
}

Johan Samsing ( $\nabla$ jsamsing@gmail.com )

Niels Bohr Institute https://orcid.org/0000-0003-0607-8741

Imre Bartos

University of Florida

Daniel D'Orazio

Niels Bohr Institute

Zoltan Haiman

Columbia University

\section{Bence Kocsis}

Rudolf Peierls Centre for Theoretical Physics

Nathan Leigh

American Museum of Natural History

Bin Liu

Cornell University

Martin Pessah

Niels Bohr Institute

Hiromichi Tagawa

Tohoku University

\section{Research Article}

Keywords: LIGO, Virgo, Chaotic 3-body Problem, Black Hole Scatterings, Disk Environments, Gravitational Wave Astrophysics

Posted Date: October 12th, 2020

DOI: https://doi.org/10.21203/rs.3.rs-89972/v1

License: (c) (1) This work is licensed under a Creative Commons Attribution 4.0 International License. Read Full License

Version of Record: A version of this preprint was published at Nature on March 9th, 2022. See the published version at https://doi.org/10.1038/s41586-021-04333-1. 



\section{Active Galactic Nuclei as Factories for Eccentric Black Hole Mergers}

3 J. Samsing ${ }^{1 *}$ I. Bartos ${ }^{2}$, D. J. D’Orazio ${ }^{1}$, Z. Haiman ${ }^{3}$, B. Kocsisis , N. W. C. Leigh ${ }^{5,6}$, B. Liu ${ }^{7}$, M.

4 E. Pessah ${ }^{1}$, H. Tagawa ${ }^{8}$.

$5^{1}$ Niels Bohr International Academy, Niels Bohr Institute, Blegdamsvej 17, 2100 Copenhagen, Denmark.

$6 \quad{ }^{2}$ Department of Physics, University of Florida, PO Box 118440, Gainesville, FL 32611, USA.

$7{ }^{3}$ Department of Astronomy, Columbia University, 550 W. 120th St., New York, NY, 10027, USA.

$8{ }^{4}$ Rudolf Peierls Centre for Theoretical Physics, Clarendon Laboratory, Parks Road, Oxford OX1 3PU, UK.

$9{ }^{5}$ Departamento de Astronomia, Universidad de Concepcion, Concepcion, Chile.

${ }^{6}$ Department of Astrophysics, AMNH, Central Park West at 79th Street, New York, NY 10024, USA.

${ }^{7}$ Department of Astronomy, Cornell University, Ithaca, NY 14853, USA.

${ }^{8}$ Astronomical Institute, Tohoku University, Sendai, Miyagi 980-8578, Japan.

Black hole mergers detected by $\mathrm{LIGO}^{1}$ and Virgo $^{2}$ continue delivering transformational dis-

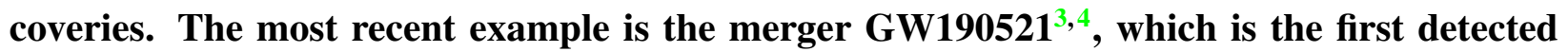
with component masses exceeding the limit predicted by stellar models ${ }^{5}$, and the first with non-zero orbital eccentricity ${ }^{6,7}$. The large masses can be explained by build up through successive mergers, which has been suggested to occur efficiently in the gas disks of active galactic nuclei $(\mathrm{AGN})^{8,9}$. The eccentricity, however, is a major puzzle. Here we show that AGN-disk environments naturally lead to a very high fraction of highly eccentric mergers, if interactions between binaries and singles are frequent ${ }^{9}$, and the interactions are constrained 
to a plane representing the AGN-disk. By deriving a statistical solution to the chaotic 3-body problem with the inclusion of General Relativistic corrections, we find in our fiducial AGNdisk model that up to $\sim 70 \%$ of all black hole mergers could appear with an eccentricity $>0.1$ in LIGO/Virgo. Besides representing the most effective mechanism for producing eccentric mergers presented to date, our results have also profound implications for the origin of GW190521, and open up new lines of research on black hole scatterings in disk environments with far-reaching implications for gravitational wave astrophysics.

Black hole mergers in the gas disks of AGN have been the focus of numerous recent studies due to multiple promising discoveries by LIGO/Virgo ${ }^{1,2}$ and due to remaining open questions ${ }^{8-12}$. Black holes that eventually merge in AGN-disks can be brought into the disk through gas-capture from the surrounding nuclear star cluster ${ }^{10}$ or can be produced through in-situ star formation ${ }^{11,13}$. Once a black hole is in the disk, it will undergo radial migration, eventually pair up with another black hole, and later merge as a result of gas-hardening and dynamical interactions with other black holes in the disk ${ }^{9}$. Recent studies show that dynamical interactions primarily between migrating binary black holes and single black holes, generally referred to as binary-single interactions, likely provide the main pathway for the last step in bringing the binaries to merger ${ }^{9,12,14-16}$. Despite progress on characterizing such interactions ${ }^{14}$, inclusion of general relativistic effects during the interactions, shown to be important in the analogous stellar-cluster case ${ }^{17,18}$, remain unexplored. Observationally, GW190521 is among the first gravitational wave sources with indications of an $\mathrm{AGN}$-disk origin ${ }^{5,19}$. It is sensible to inquire whether its non-zero eccentricity ${ }^{6,7}$ is highly unlikely from an astrophysical perspective or if it could arise naturally as a distinct signature characteristic 


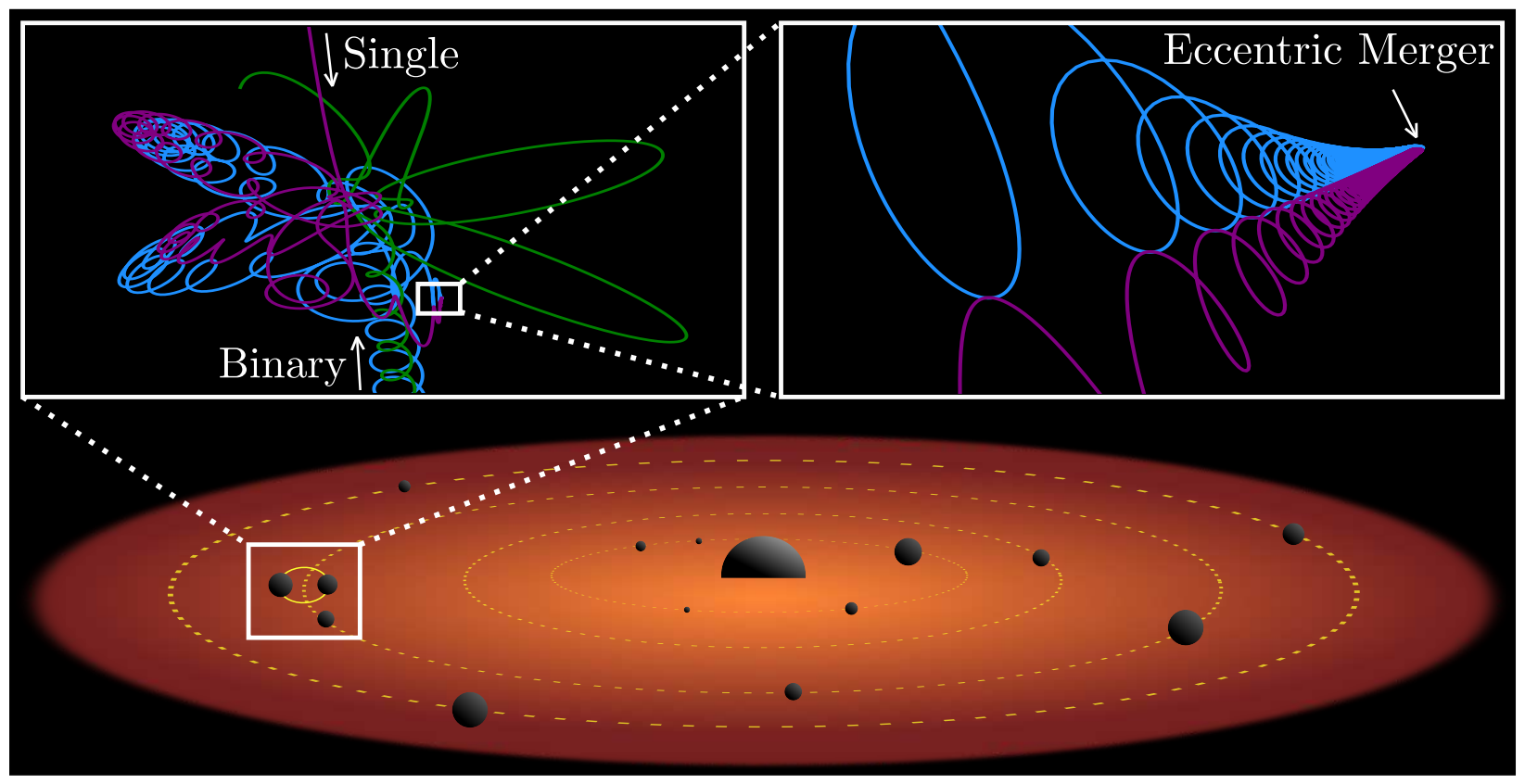

Figure 1: Illustration of an eccentric LIGO/Virgo source forming in an AGN-disk. Bottom: AGN-disk (not to scale) with its central super-massive black hole, and a population of smaller orbiting black holes. These smaller black holes occasionally pair-up to form binary black holes, which often undergo scatterings with the single black hole population. Top: Outcome of a $\left[50 M_{\odot}, 80 M_{\odot}\right]$ binary black hole interacting with an incoming $\left[70 M_{\odot}\right]$ black hole that results in a $\left[80 M_{\odot}, 70 M_{\odot}\right]$ binary black hole merger during the interaction (3-body merger) with an eccentricity $\sim 0.5$ in LIGO/Virgo. When the dynamical effects of gravitational wave emission are included, and interactions are constrained to a 2-dimensional disk plane, we find a majority population of such eccentric LIGO/Virgo sources. This also provides a possible explanation for GW190521. 
of dynamically induced AGN-disk mergers.

With this motivation, we here present the first estimates of how binary black holes merge through binary-single interactions in AGN-disk environments when the dynamical effect of gravitational wave emission is included via the 2.5 -post-Newtonian $(2.5 \mathrm{PN})$ correction term in the $N$-body equations-of-motion ${ }^{20}$. As a first step, we consider a thin disk model where interactions are constrained to a 2-dimensional (2D)-plane representing the AGN-disk. In this case, we find that for mass-, length-, and time-scales relevant for AGN-disk environments, the probability that two of the black holes merge while the three are interacting (see Fig. 1), denoted a 3-body merger, is generally greater than the probability that this pair instead survives the interaction and then merges before undergoing its next interaction, denoted a 2-body merger. As the 3-body mergers generally have very high characteristic eccentricities ${ }^{21}$, a remarkable result of this is that binary black hole mergers with an eccentricity $>0.1$ in the LIGO/Virgo bands could be as frequent as standard 'circular' mergers from this channel. This is in stark contrast to the spherical cluster case, where only a few percent of 3-dimensional (3D) isotropic scatterings produce 3-body mergers ${ }^{18}$. Our results not only provide the first explanation for why GW190521 would naturally appear in the LIGO/Virgo frequency band with a measurable eccentricity if it did form in an AGN-disk ${ }^{19}$, but also opens up a rich new line of research on dynamically modeling the formation of binary black hole mergers in disk environments. Below we present our results by first deriving the merger probability and then the fraction of mergers that will manifest as eccentric sources in the LIGO/Virgo band. Our analytical solutions are restricted to the equal-mass case, but we perform several full 2.5-PN-simulations to explore the more likely unequal-mass case. One example is shown in Fig. 1, which illustrates a 
possible origin for GW190521.

Merger Probability. To estimate the relative occurrence rates of 3-body and 2-body mergers, we start by calculating the probability that a dynamically assembled binary black hole merges within some timescale $\tau$, denoted by $P\left(t_{G W}<\tau\right)$, where $t_{G W}$ is here the initial merger time. The time $t_{G W}$ for an eccentric binary black hole is given by ${ }^{22}$,

$$
t_{G W} \approx \frac{5 c^{5}}{512 G^{3}} \frac{a^{4}}{m^{3}}\left(1-e^{2}\right)^{7 / 2} \approx t_{c} \times\left(1-e^{2}\right)^{7 / 2}
$$

where $e, a$, and $m$ are the initial orbital eccentricity, semi-major axis, and individual black hole mass, respectively. The eccentricities of binary black holes dynamically assembled through coplanar interactions that are isotropic in 2D are distributed according to ${ }^{23,24}$,

$$
P(e) \approx e / \sqrt{1-e^{2}}
$$

from which it directly follows that

$$
P\left(t_{G W}<\tau\right) \approx\left(\tau / t_{c}\right)^{1 / 7}
$$

where we have used that $P\left(e>e_{0}\right)=\sqrt{\left(1-e_{0}^{2}\right)}$. With this expression, we estimate the probability that a binary black hole with given orbital parameters will merge through a 2-body or a 3-body merger process. The probability of a 2-body merger, $p_{2}$, is found by equating $\tau$ in Eq. 3 with the time until the binary black hole undergoes its next interaction, $t_{\mathrm{int}}$,

$$
p_{2} \approx\left(t_{\mathrm{int}} / t_{c}\right)^{1 / 7} \approx 0.07\left[\frac{t_{\mathrm{int}}}{10^{5} \mathrm{yr}}\right]^{1 / 7}\left[\frac{m}{20 M_{\odot}}\right]^{3 / 7}\left[\frac{a}{1 \mathrm{AU}}\right]^{-4 / 7}
$$


where we have normalized to values characteristic of AGN-disk environments ${ }^{9}$. To estimate the 3-body merger probability, $p_{3}$, we start by describing the often highly chaotic binary-single interaction as a series of $\mathcal{N}$ temporary states, each characterized by a binary black hole with a bound $\operatorname{single}^{21}$ (Fig. 1, left panel). For one of these temporary binary black holes to merge, its inspiral time $t_{G W}$ must be smaller than a characteristic timescale for the system ${ }^{25,26}, T_{0} \sim \sqrt{a^{3} /(G m)}$. Hence, the probability that a binary black hole undergoes a 3-body merger during a single interaction, is found by equating $\tau$ in Eq. 3 with $T_{0}$,

$$
p_{3} \approx \mathcal{N} \times\left(T_{0} / t_{c}\right)^{1 / 7} \approx 0.15\left[\frac{m}{20 M_{\odot}}\right]^{5 / 14}\left[\frac{a}{1 \mathrm{AU}}\right]^{-5 / 14},
$$

which also can be expressed in more fundamental terms as $p_{3} \approx \mathcal{N} \times\left(v_{\text {orb }} / c\right)^{5 / 7}$, where $v_{\text {orb }}$ is the binary orbital velocity. Here and throughout the paper we use $\mathcal{N}=20$, as found numerically ${ }^{18}$. Note that the exact factor for $T_{0}$, which sets the characteristic 3-body timescale, is not important due to the $1 / 7$ power suppression.

From the ratio $p_{3} / p_{2}=\mathcal{N} \times\left(T_{0} / t_{\text {int }}\right)^{1 / 7} \propto t_{\text {int }}^{-1 / 7} m^{-1 / 14} a^{3 / 14}$, it follows that for large enough $a$, the probability for a 3-body merger exceeds that of a 2-body merger. The $a$ at which the two probabilities are equal, denoted here by $a_{32} \equiv a\left(p_{3} / p_{2}=1\right)$, is

$$
a_{32} \approx \mathcal{N}^{-14 / 3} t_{\mathrm{int}}^{2 / 3} G^{1 / 3} m^{1 / 3} \approx 0.01 \sim 0.1 \mathrm{AU}\left[\frac{t_{\mathrm{int}}}{10^{5} \mathrm{yr}}\right]^{2 / 3}\left[\frac{m}{20 M_{\odot}}\right]^{1 / 3} .
$$

That is, for $a \gtrsim a_{32}, 3$-body mergers dominate over 2-body mergers. The steep, $-14 / 3$ dependence on $\mathcal{N}$ introduces some uncertainty to this analytical estimate, as indicated by the interval $0.01 \sim$ 0.1 AU in the last equality. State-of-the-art work ${ }^{9}$ indicates that binary-single scatterings become important for $a \lesssim 10 \mathrm{AU}$. Hence, this derivation provides the first strong argument that 3-body 


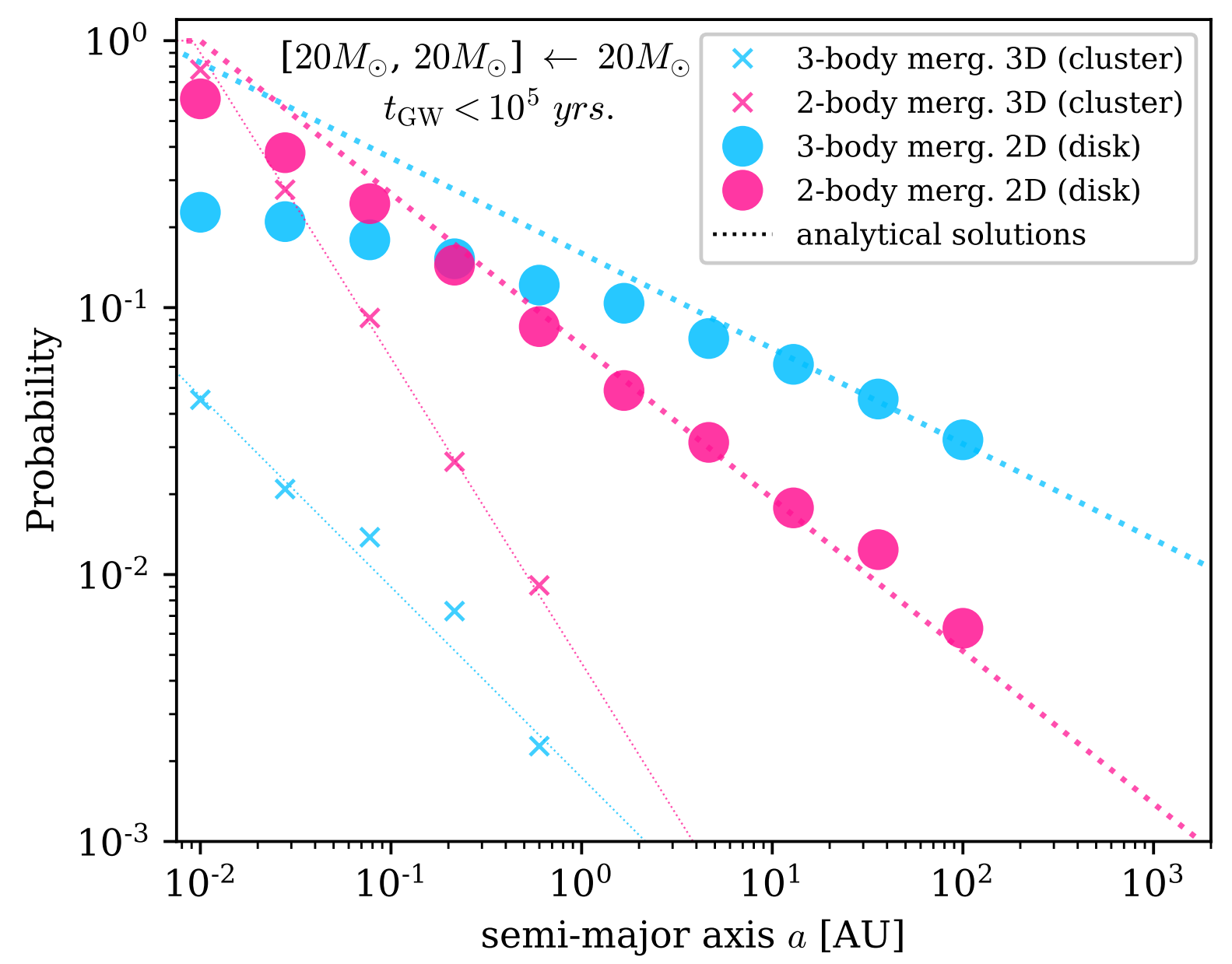

Figure 2: Merger probability. Probability of undergoing a 2-body merger (red) or a 3-body merger (blue) from binary-single interactions, as a function of initial semi-major axis, $a$, of the target binary. All three interacting black holes have the same mass $m=20 M_{\odot}$, and the merger time-limit is set to $t_{G W}<t_{\mathrm{int}}=10^{5} \mathrm{yr}$. Circles: Numerical results from 2D interactions (disk). Crosses: Numerical results from 3D interactions (cluster). Lines: Analytical solutions. Numerical results are obtained using full 2.5-PN-simulations, and analytical solutions are outlined in this paper. 
mergers could play a key-role in mediating binary black hole mergers in AGN-disk environments.

Fig. 2 shows $p_{2}$ and $p_{3}$ as a function of $a$, derived using full 2.5-PN-simulations ${ }^{25}$, assuming isotropic interactions in 2D (disk) or 3D (cluster). As the figure shows, the probabilities derived from Eq. 4 and Eq. 5 agree with the simulations for large $a$, but start to deviate when $p_{2}+p_{3}$ approaches unity. The reason for this has at least two explanations: First, for assembling a 3-body merger it is necessary to enter a resonant state, where the single is not promptly ejected. However, only a limited number of such states are available ${ }^{27}$, and $p_{3}$ will therefore have to asymptote to a value $<1$ for small $a$. This is illustrated in Fig. 3, where 'areas of regularity' (single-color regions) and 'areas of chaos' (coarse-grained regions) indicate, respectively, prompt ejections and resonating states. Second, our analytical solution from Eq. 5 assumes that $p_{3}$ can be written out as an uncorrelated sum over the merger probabilities per temporary binary black hole. However, this approximation naturally breaks down when $p_{3}$ approaches $1^{28}$. Despite these technical details, it clearly follows from Fig. 2 that $p_{3} \gtrsim p_{2}$ for $a \gtrsim 0.1 \mathrm{AU}$, as we also argued analytically. Fig. 2 also shows the probability for 2-body and 3-body mergers assuming 3D isotropic scatterings, denoted here by $p_{2}^{(3 \mathrm{D})}$ and $p_{3}^{(3 \mathrm{D})}$, respectively. In this case, the eccentricity distribution $P(e)$ instead follows ${ }^{29} P(e)=2 e$, from which one finds ${ }^{18}$ that $p_{3}^{(3 \mathrm{D})} \approx \mathcal{N} \times\left(T_{0} / t_{c}\right)^{2 / 7}$. The fractional difference in going from the 3D-cluster case to our considered 2D-disk case, is therefore $p_{3} / p_{3}^{(3 \mathrm{D})} \approx\left(T_{0} / t_{c}\right)^{-1 / 7} \approx 10^{2} \times\left[m / 20 M_{\odot}\right]^{-5 / 14}[a / \mathrm{AU}]^{5 / 14}$, which illustrates why AGN-disk environments can lead to a major enhancement in eccentric LIGO/Virgo sources. From Fig. 3, it also follows that 2-body and 3-body mergers form effectively from both positive and negative $b^{\prime}$, i.e. counter-rotating and co-rotating interactions, respectively. That is, our conclusions do not strongly 
depend on how the binary black hole rotates relative to the AGN-disk and the incoming single black hole.

We have computed the probability for 2-body and 3-body mergers to occur during one binarysingle scattering. However, a binary black hole is often driven to merger over multiple scatterings ${ }^{18}$. To determine whether mergers typically occur in the 2-body or the 3-body dominated regime, we now compare the semi-major axis above which 3-body mergers dominate $\left(a_{32} \sim 0.1 \mathrm{AU}\right)$ to the typical semi-major axis for which the merger probability over multiple interactions approaches unity ( $a_{m}$ below). For this, we consider a model where a binary black hole starts with semi-major axis $a_{i}$, and subsequently undergoes binary-single scatterings, each of which decreases its semimajor axis by a fraction $\delta$, until it reaches a lower value of $a_{f}<a_{i}{ }^{14,18}$. The value of $\delta$ can be shown to equal ${ }^{30} \delta=1-\gamma^{-1}$, where $\gamma$ relates to the distribution in binary binding energy ${ }^{14,29}$, as $P\left(E_{b}\right) \propto E_{b}^{-\gamma}$. For co-planar scatterings ${ }^{23} \gamma=3$, which implies that $\delta \approx 2 / 3$. Assuming the binary black hole is being scattered from large $a_{i}$ where $p_{3}>p_{2}$, then the total integrated probability for the binary black hole to have undergone a gravitational wave merger during one of its scatterings from $a_{i}$ to $a_{f}$ is given by ${ }^{18}$,

$$
P_{3}\left(a_{f}\right) \approx \frac{1}{1-\delta} \int_{a_{i}}^{a_{f}} \frac{p_{3}(a)}{a} d a \approx \frac{42}{5} p_{3}\left(a_{f}\right)
$$

where we have assumed that $a_{i} \gg a_{f}$. In this model, the semi-major axis for which $a\left(P_{3}=1\right)$, denoted here by $a_{m}$, represents the minimum possible value for $a$. Using the above relations we find,

$$
a_{m} \approx 1 \mathrm{AU} \times\left[\frac{m}{20 M_{\odot}}\right]
$$




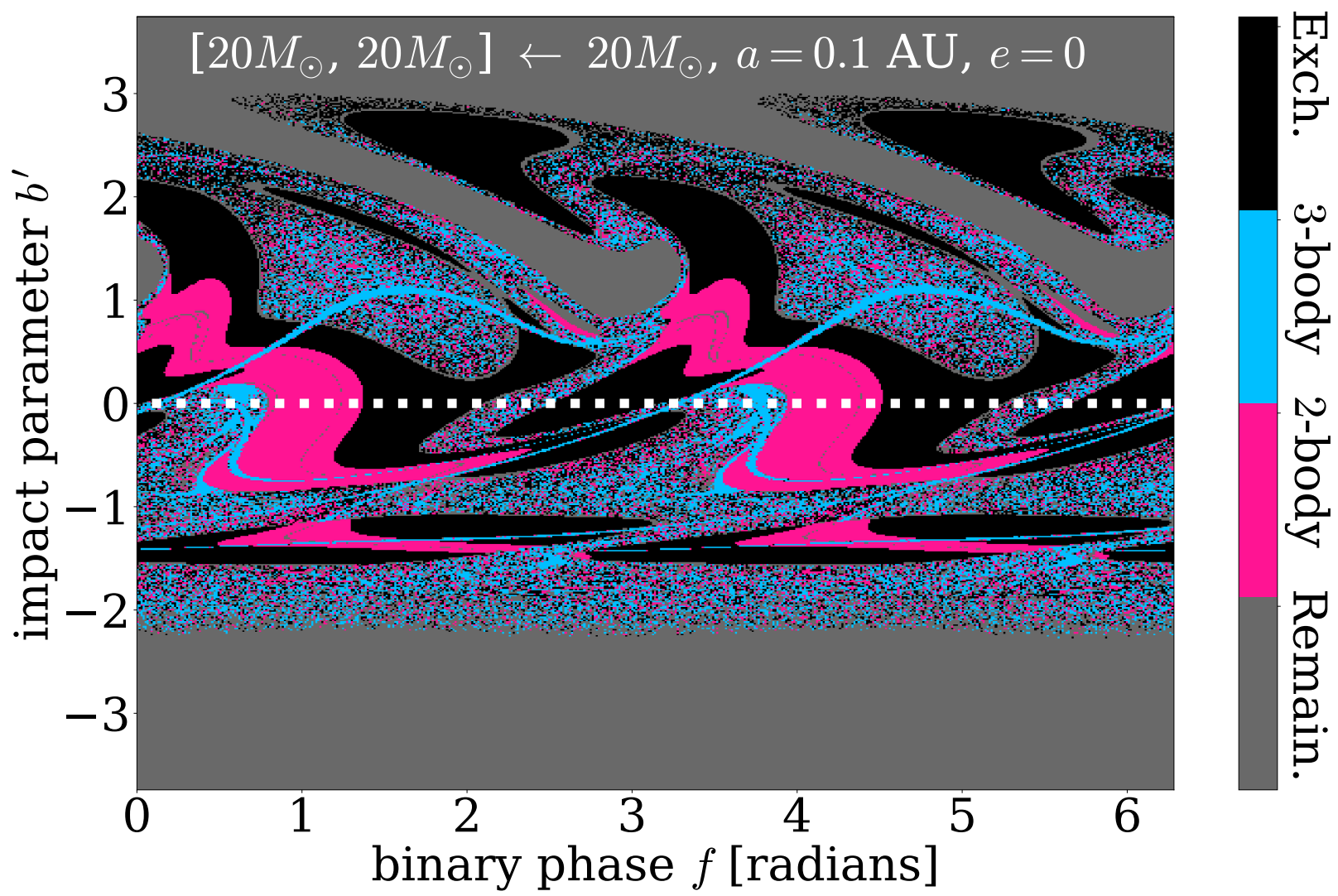

Figure 3: Phase-space distribution of outcomes. Results from co-planar scatterings between a binary and a single black hole with $m=20 M_{\odot}$, as a function of binary phase $f$, and impact parameter $b^{\prime}$. Here, $f$ is measured relative to $b^{\prime}=0$ at the time the single is at a distance $20 a$ from the binary, and $b^{\prime}=\left(b_{\infty} / a\right)\left(v_{\infty} / v_{c}\right)$, where $b_{\infty}, v_{\infty}, a$, and $v_{c}$ are the impact parameter at infinity, the relative velocity at infinity, the initial binary semi-major axis, and the critical binary velocity $v_{c}^{2}=(3 / 2) G m / a$, respectively. Black: Exchange interactions not leading to a merger. Blue: Interactions leading to a 3-body merger. Red: Interactions leading to a 2-body merger. Grey: Remaining set. The merger time-limit is set to $t_{G W}<t_{\text {int }}=10^{5}$ yr. In our setup, the binary black hole and the incoming black hole are co-rotating and counter-rotating above and below the white dotted horizontal line, respectively. 
which clearly is larger than both $a_{32}$ from Eq. 6, and recently reported values of the semi-major axis before merger ${ }^{9}$. Despite the simplicity of this model, this suggests that 3-body mergers are able to dominate the rate of dynamically assembled binary black hole mergers in 2D disk environments. Below we illustrate the major observational consequences of this finding.

Eccentric LIGO/Virgo Sources. Having argued that 3-body mergers could dominate the binary black hole merger rate in AGN-disks, we now address the associated observational implications. One immediate possibility is a non-negligible population of binary black hole mergers that appear with a detectable eccentricity, $e_{f}$, at some gravitational wave frequency, $f_{G W}$; a population that we loosely refer to as 'eccentric sources'. For a binary black hole to appear with an eccentricity $e>e_{f}$ at gravitational wave peak frequency $f_{G W} \approx \pi^{-1} \sqrt{2 G m / r_{f}^{3}}$, where $r_{f}$ is here the pericenter distance, the initial binary black hole peri-center distance at assembly (or 'capture') has to be smaller than $r_{c}\left(e_{f}\right)$, where $r_{c}\left(e_{f}\right)$ is given by ${ }^{18,31}$,

$$
r_{c}\left(e_{f}\right) \approx\left(\frac{2 G m}{f_{G W}^{2} \pi^{2}}\right)^{1 / 3} \frac{1}{2} \frac{1+e_{f}}{e_{f}^{12 / 19}}\left[\frac{425}{304}\left(1+\frac{121}{304} e_{f}^{2}\right)^{-1}\right]^{870 / 2299}
$$

Therefore, the probability for a binary-single interaction to result in an eccentric source followed by merger, is to leading order the probability that two of the three black holes form a temporary binary black hole with an initial peri-center $r_{0}<r_{c}\left(e_{f}\right)$. To calculate this probability, denoted by $p_{e c c}$, where the subscript $e c c$ refers to 'eccentric', we use that $P\left(e>e_{0}\right) \approx \sqrt{2\left(1-e_{0}\right)}$ in the high eccentricity limit, together with the Keplerian relation $r_{0} / a=1-e_{0}$, from which it directly follows that ${ }^{18}$,

$$
p_{e c c} \approx \mathcal{N} \times \sqrt{\frac{2 r_{c}\left(e_{f}\right)}{a}}
$$


For values relevant for LIGO/Virgo ${ }^{32}$,

$$
p_{e c c}(e>0.1: 10 \mathrm{~Hz}) \approx 0.15\left[\frac{m}{20 M_{\odot}}\right]^{1 / 6}\left[\frac{a}{1 \mathrm{AU}}\right]^{-1 / 2}
$$

where this relation includes all sources that will appear with $e>0.1$ at $10 \mathrm{~Hz}$ or above. By now performing an integration similar to Eq. 7, one finds that the total probability that a binary black hole undergoes an eccentric merger during scatterings from $a_{i}$ to $a_{f}$ evaluates to $P_{\text {ecc }}\left(a_{f}\right) \approx$ $6 p_{e c c}\left(a_{f}\right)$. The ratio of the number of eccentric sources to all 3-body mergers is therefore $P_{\text {ecc }} / P_{3} \approx$ $(5 / 7)\left(p_{e c c} / p_{3}\right)$. As $p_{e c c}$ is generally $<p_{3}$, this finally leads us to the inequality,

$$
P_{e c c}<\frac{5}{7} P_{3}
$$

which states that up to $5 / 7 \sim 70 \%$ of all mergers will appear as eccentric LIGO/Virgo sources in this model.

To illustrate that eccentric binary black hole mergers are not a unique outcome of equalmass interactions, we also consider results from full 2.5-PN-scatterings between a binary black hole $\left[40 M_{\odot}, 20 M_{\odot}\right]$ with $a=1 \mathrm{AU}$ and an incoming single black hole [20M $M_{\odot}$. Fig. 4 (left panel) shows the gravitational wave peak frequency distribution at the time of formation (assembly) for each binary black hole that eventually merges. Nearly all 3-body mergers form near or in the

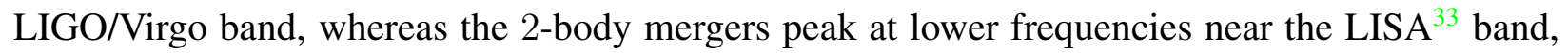
as naturally follows from the difference in characteristic time scales ${ }^{34,35}$. Fig. 4 (right panel) shows the corresponding cumulative eccentricity distribution at $10 \mathrm{~Hz}$, where we have used the quadrupole approximation $^{22}$ for propagating the sources from their initial gravitational wave peak frequency. In agreement with our analytical calculations, we see that most of the binary black hole 

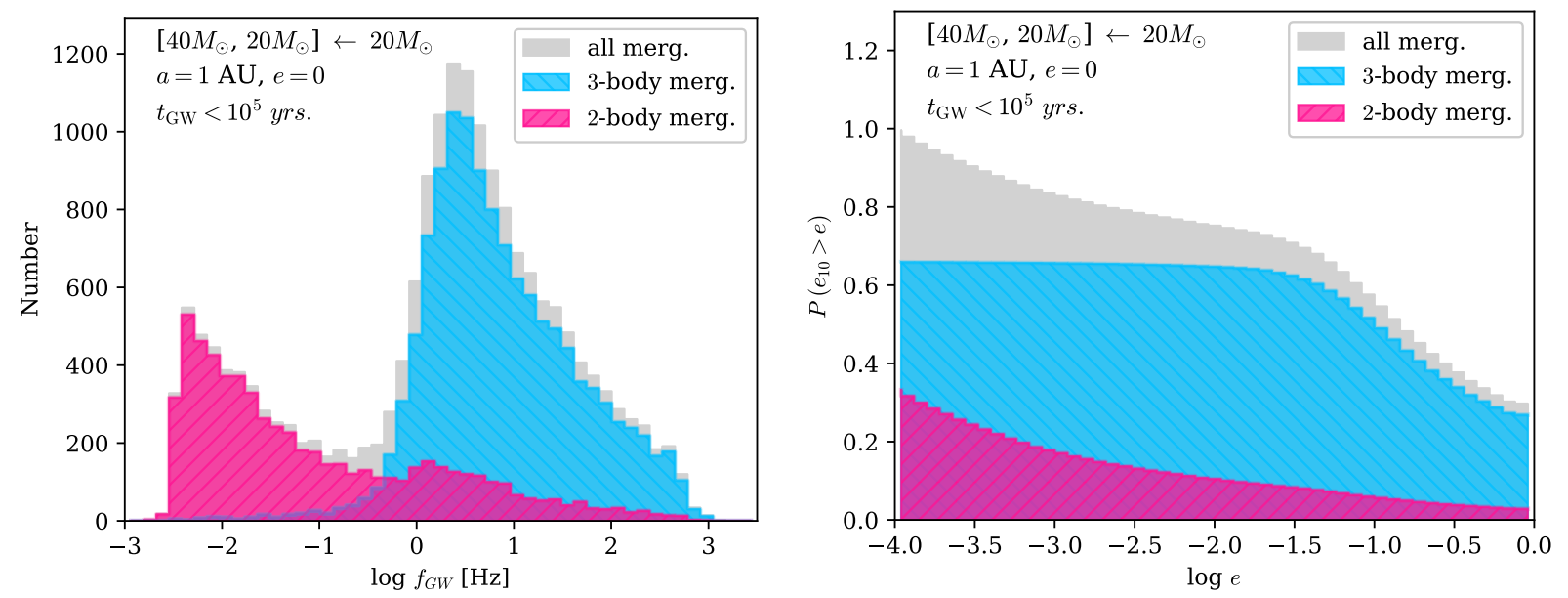

Figure 4: Eccentricity and gravitational wave frequency distributions. Results from $10^{5}$ independent co-planar 2.5-PN-scatterings, between an incoming $20 M_{\odot}$ black hole and a $\left[40 M_{\odot}, 20 M_{\odot}\right]$ binary black hole with an initial semi-major axis and eccentricity of $a=1 \mathrm{AU}$ and $e=0$, respectively. The red and blue distributions show the outcome from 2-body mergers and 3-body mergers, respectively, where grey is the total number. We only include binary black holes with resultant inspiral times of $t_{G W}<t_{\text {int }}=10^{5} \mathrm{yr}$. Left: Distribution of binary black hole gravitational wave peak frequency, $f_{G W}$, measured at the time of assembly. Right: Cumulative distribution of binary black hole orbital eccentricities propagated to $10 \mathrm{~Hz}$. For sources formed with $f_{G W}>10 \mathrm{~Hz}$, the corresponding eccentricity has been set to $e=1$. 
mergers have an eccentricity $e>0.1$ at $f_{G W}>10 \mathrm{~Hz}$ or above. Note that sources forming in band, will generally appear as burst sources ${ }^{36}$, and some will even remain eccentric close to merger.

Conclusions. Orbital eccentricity constitutes one out of only three intrinsic parameter-sets that can be directly deduced from an observed gravitational wave signal, where the other two are black hole masses and spins. The rate of eccentric sources is typically expected to be relatively low, but every such source provides invaluable information into the astrophysical formation mechanism of gravitational wave mergers. For example, eccentric mergers naturally form in galactic nuclei during gravitational wave capture encounters ${ }^{37-39}$, in globular clusters during multi-body interactions $^{17,18}$, and in the field through Lidov-Kozai oscillations ${ }^{40}$, but essentially never through standard isolated binary evolution. A true milestone on the observational level is GW190521, which is the first gravitational wave source consistent with having a non-zero eccentricity ${ }^{6,7}$. Its relatively high-mass components, high misaligned spin and possible associated electromagnetic flare $^{19}$ suggest a possible AGN-disk origin ${ }^{19}$, but the additional non-zero eccentricity has been a major puzzle.

In this paper we have shown that AGN-disks provide a natural environment for efficiently producing eccentric LIGO/Virgo sources, if the binaries are primarily driven to merger through scatterings with singles in the plane of the disk ${ }^{9}$. In particular, by deriving a statistical solution to the general relativistic three-body problem, we prove that such $2 \mathrm{D}$ planar (disk) scatterings generally produce eccentric sources at a rate that is orders-of-magnitude higher than what is found in the analogue 3D isotropic case (cluster). This not only provides the first possible explanation 
for the non-zero eccentricity of GW190521, but also opens up a new area of research with the potential to play a key role in probing the origin of gravitational wave sources. In upcoming studies we will include our framework in state-of-the-art AGN-disk models, and explore the role of gas-drag, deviations from our simple 2D disk description, the secular evolution of hierarchical systems, and how possible joint electromagnetic and gravitational wave observations could be used to constrain the physical properties of the AGN-disk channel. 
Acknowledgements The authors are grateful to Nirban Bose, Kelley Holley-Bockelmann, Archana Pai, Mike Zevin and M. Safarzadeh for their useful suggestions. J.S. is supported by the European Unions Horizon 2020 research and innovation programme under the Marie Sklodowska-Curie grant agreement No. 844629. D.J.D. acknowledges support of a research grant from Villum Fonden. I.B. acknowledges support from the Alfred P. Sloan Foundation. H.T. is financially supported by the Grants-in-Aid for Basic Research by the Ministry of Education, Science and Culture of Japan (HT:17H01102, 17H06360). N.W.C.L. gratefully acknowledges support from the Chilean government via Fondecyt Iniciación Grant \#11180005. Z.H. acknowledges support from NASA grant NNX15AB19G and NSF grants AST-1715661 and AST-2006176. This project has received funding from the European Research Council (ERC) under the European Union's Horizon 2020 research and innovation programme ERC-2014-STG under grant agreement No 638435 (GalNUC) to BK.

Author contributions J.S. led the work, carried out the simulations and calculations, and wrote the initial manuscript together with I.B. and D.D. All authors contributed to the intellectual development of the ideas and the preparation of the final manuscript.

Competing Interests The authors declare no competing financial interests.

Correspondence Correspondence and requests for materials should be addressed to J. Samsing (email: jsamsing@gmail.com) 
1. Aasi, J. et al. Advanced LIGO. CQG 32, 074001 (2015).

2. Acernese, F. et al. Advanced Virgo: a second-generation interferometric gravitational wave detector. CQG 32, 024001 (2015).

3. Abbott, R., Abbott, T. D., Abraham, S. et al. GW190521: A Binary Black Hole Merger with a Total Mass of $150 \mathrm{M}_{\odot}$. Phys. Rev. Lett. 125, 101102 (2020).

4. Abbott, R., Abbott, T. D., Abraham, S. et al. Properties and astrophysical implications of the 150 Msun binary black hole merger GW190521. arXiv e-prints arXiv:2009.01190 (2020). 2009.01190.

5. Abbott, R. et al. Properties and astrophysical implications of the $150 \mathrm{~m} \odot$ binary black hole merger GW190521. Astrophys. J. Lett. 900, L13 (2020). URL https : / / doi . org/ 10 . $3847 \div 2 \mathrm{~F} 2041-8213 \div 2 \mathrm{Faba} 493$.

6. Gayathri, V. et al. GW190521 as a Highly Eccentric Black Hole Merger. arXiv e-prints arXiv:2009.05461 (2020). 2009.05461.

7. Romero-Shaw, I. M., Lasky, P. D., Thrane, E. \& Calderon Bustillo, J. GW190521: orbital eccentricity and signatures of dynamical formation in a binary black hole merger signal.

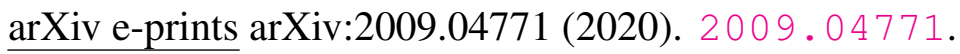

8. McKernan, B. et al. Constraining Stellar-mass Black Hole Mergers in AGN Disks Detectable with LIGO. Astrophys. J. 866, 66 (2018). 1702 . 07818. 
9. Tagawa, H., Haiman, Z. \& Kocsis, B. Formation and Evolution of Compact-object Binaries in AGN Disks. Astrophys. J. 898, 25 (2020). 1912.08218.

10. Bartos, I., Kocsis, B., Haiman, Z. \& Márka, S. Rapid and Bright Stellar-mass Binary Black Hole Mergers in Active Galactic Nuclei. Astrophys. J. 835, 165 (2017). 1602.03831.

11. Stone, N. C., Metzger, B. D. \& Haiman, Z. Assisted inspirals of stellar mass black holes embedded in AGN discs: solving the 'final au problem'. MNRAS 464, 946-954 (2017). 1602.04226.

12. Secunda, A. et al. Orbital Migration of Interacting Stellar Mass Black Holes in Disks around Supermassive Black Holes. Astrophys. J. 878, 85 (2019). 1807.02859.

13. Cantiello, M., Jermyn, A. S. \& Lin, D. N. C. Stellar Evolution in AGN Disks. arXiv e-prints arXiv:2009.03936 (2020). 2009.03936.

14. Leigh, N. W. C. et al. On the rate of black hole binary mergers in galactic nuclei due to dynamical hardening. MNRAS 474, 5672-5683 (2018). 1711.10494.

15. Tagawa, H., Haiman, Z., Bartos, I. \& Kocsis, B. Spin Evolution of Stellar-mass Black Hole Binaries in Active Galactic Nuclei. Astrophys. J. 899, 26 (2020). 2004.11914.

16. Kocsis, B., Gáspár, M. E. \& Márka, S. Detection Rate Estimates of Gravity Waves Emitted during Parabolic Encounters of Stellar Black Holes in Globular Clusters. Astrophys. J. 648, 411-429 (2006). astro-ph/ 0603441. 
17. Rodriguez, C. L. et al. Post-Newtonian dynamics in dense star clusters: Formation, masses, and merger rates of highly-eccentric black hole binaries. Phys. Rev. D 98, 123005 (2018). 1811.04926.

18. Samsing, J. Eccentric black hole mergers forming in globular clusters. Phys. Rev. D 97, 103014 (2018). 1711.07452.

19. Graham, M. J. et al. Candidate Electromagnetic Counterpart to the Binary Black Hole Merger Gravitational-Wave Event S190521g*. Phys. Rev. Lett. 124, 251102 (2020). 2006.14122.

20. Blanchet, L. Gravitational Radiation from Post-Newtonian Sources and Inspiralling Compact Binaries. Living Reviews in Relativity 9 (2006).

21. Samsing, J., MacLeod, M. \& Ramirez-Ruiz, E. The Formation of Eccentric Compact Binary Inspirals and the Role of Gravitational Wave Emission in Binary-Single Stellar Encounters. Astrophys. J. 784, 71 (2014). 1308.2964.

22. Peters, P. Gravitational Radiation and the Motion of Two Point Masses. Phys. Rev. 136, B1224-B1232 (1964).

23. Valtonen, M. \& Karttunen, H. The Three-Body Problem (2006).

24. Stone, N. C. \& Leigh, N. W. C. A statistical solution to the chaotic, non-hierarchical threebody problem. Nature 576, 406-410 (2019). 1909.05272.

25. Samsing, J., MacLeod, M. \& Ramirez-Ruiz, E. Formation of Tidal Captures and Gravitational Wave Inspirals in Binary-single Interactions. Astrophys. J. 846, 36 (2017). 1609.09114. 
26. Samsing, J., MacLeod, M. \& Ramirez-Ruiz, E. Dissipative Evolution of Unequal-mass Binary-single Interactions and Its Relevance to Gravitational-wave Detections. Astrophys. J. 853, 140 (2018). 1706.03776.

27. Samsing, J. \& Ilan, T. Topology of black hole binary-single interactions. MNRAS 476, 15481560 (2018). 1706.04672.

28. Samsing, J. et al. Probing the black hole merger history in clusters using stellar tidal disruptions. Phys. Rev. D 100, 043009 (2019). 1901.02889.

29. Heggie, D. C. Binary evolution in stellar dynamics. MNRAS 173, 729-787 (1975).

30. Samsing, J. \& Hotokezaka, K. Populating the Black Hole Mass Gaps In Stellar Clusters: General Relations and Upper Limits. arXiv e-prints arXiv:2006.09744 (2020). 2006.09744.

31. Samsing, J., Askar, A. \& Giersz, M. MOCCA-SURVEY Database. I. Eccentric Black Hole Mergers during Binary-Single Interactions in Globular Clusters. Astrophys. J. 855, 124 (2018). 1712.06186.

32. Gondán, L. \& Kocsis, B. Measurement Accuracy of Inspiraling Eccentric Neutron Star and Black Hole Binaries Using Gravitational Waves. Astrophys. J. 871, 178 (2019). 1809. 00672.

33. Amaro-Seoane, P. et al. Laser Interferometer Space Antenna. ArXiv e-prints (2017). 1702. 00786. 
34. Samsing, J. \& D’Orazio, D. J. Black Hole Mergers From Globular Clusters Observable by LISA I: Eccentric Sources Originating From Relativistic N-body Dynamics. MNRAS (2018). 1804.06519.

35. D’Orazio, D. J. \& Samsing, J. Black hole mergers from globular clusters observable by LISA II. Resolved eccentric sources and the gravitational wave background. MNRAS 481, 47754785 (2018). 1805.06194.

36. Loutrel, N. Analytic waveforms for eccentric gravitational wave bursts. Classical and Quantum Gravity 37, 075008 (2020). 1909.02143.

37. O’Leary, R. M., Kocsis, B. \& Loeb, A. Gravitational waves from scattering of stellar-mass black holes in galactic nuclei. MNRAS 395, 2127-2146 (2009). 0807.2638.

38. Gondán, L., Kocsis, B., Raffai, P. \& Frei, Z. Eccentric Black Hole Gravitational-wave Capture Sources in Galactic Nuclei: Distribution of Binary Parameters. Astrophys. J. 860, 5 (2018). 1711.09989.

39. Rasskazov, A. \& Kocsis, B. The Rate of Stellar Mass Black Hole Scattering in Galactic Nuclei. Astrophys. J. 881, 20 (2019). 1902.03242.

40. Liu, B., Lai, D. \& Wang, Y.-H. Black Hole and Neutron Star Binary Mergers in Triple Systems. II. Merger Eccentricity and Spin-Orbit Misalignment. Astrophys. J. 881, 41 (2019). 1905. 00427. 


\section{Figures}

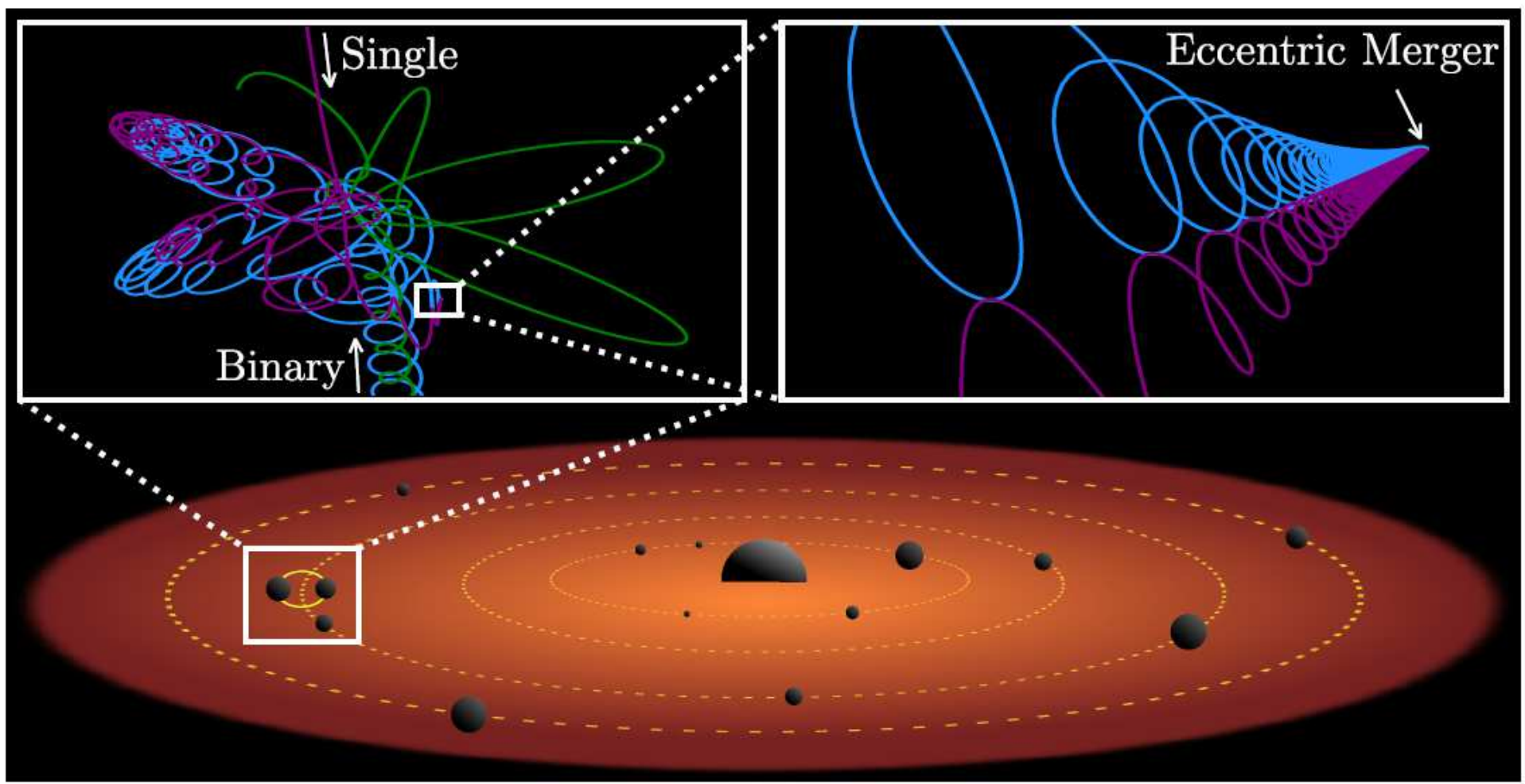

\section{Figure 1}

Illustration of an eccentric LIGO/Virgo source forming in an AGN-disk. Bottom: AGN-disk (not to scale) with its central super-massive black hole, and a population of smaller orbit-ing black holes. These smaller black holes occasionally pair-up to form binary black holes, which often undergo scatterings with the single black hole population. Top: Outcome of a [50M, $80 \mathrm{M}]$ binary black hole interacting with an incoming [70M] black hole that results in a [80M, 70M] binary black hole merger during the interaction (3body merger) with an eccentricity $\otimes 0.5$ in LIGO/Virgo. When the dynamical effects of gravitational wave emission are included, and in-teractions are constrained to a 2-dimensional disk plane, we find a majority population of such eccentric LIGO/Virgo sources. This also provides a possible explanation for GW190521. 


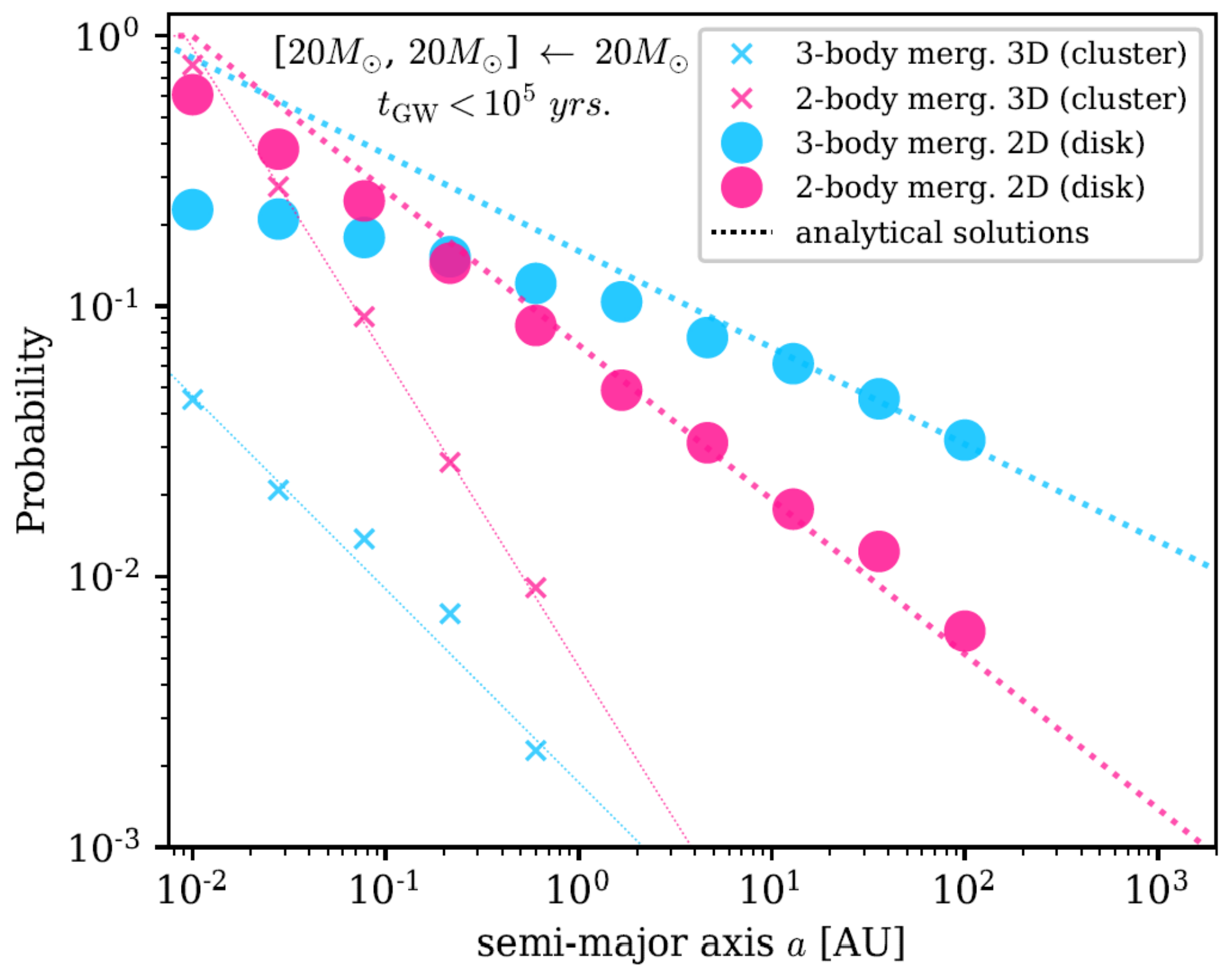

Figure 2

Probability of undergoing a 2-body merger (red) or a 3-body merger (blue) from binary-single interactions, as a function of initial semi-major axis, a, of the target binary. All three interacting black holes have the same mass $m=20 \mathrm{M}$, and the merger time-limit is set to $\mathrm{tGW}<\mathrm{tint}=105 \mathrm{yr}$. Circles: Numerical results from 2D interactions (disk). Crosses: Numerical results from 3D interactions (cluster). Lines: Analytical solutions. Numerical results are obtained using full 2.5-PN-simulations, and analytical solutions are outlined in this paper. 


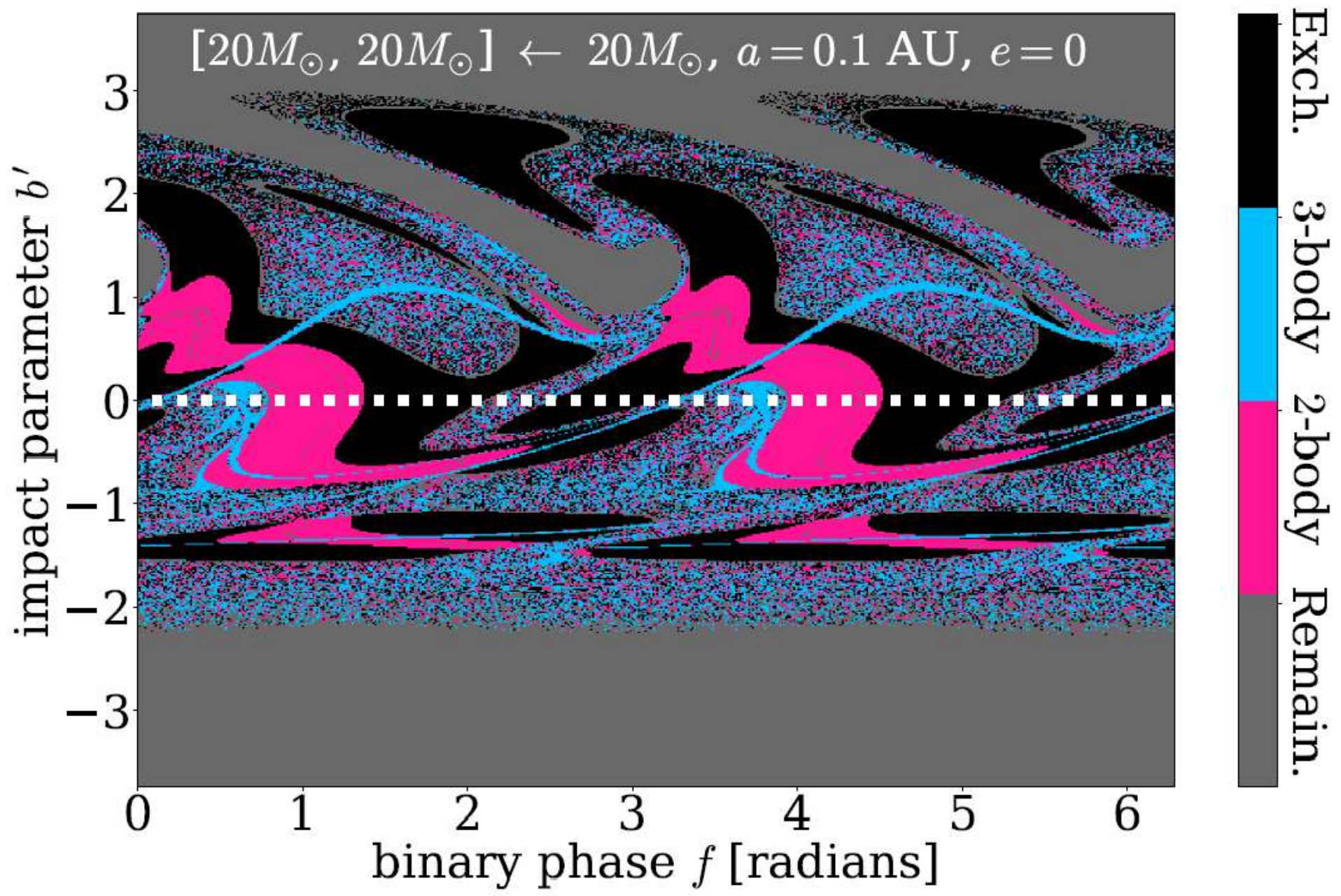

Figure 3

Phase-space distribution of outcomes. Results from co-planar scatterings between a binary and a single black hole with $m=20 M$, as a function of binary phase $f$, and impact parameter $b$. Here, $f$ is measured relative to $b=0$ at the time the single is at a distance $20 a$ from the binary, and $b=(b \infty / a)(v \infty / v c)$, where $b \infty, v \infty, a$, and $v c$ are the impact parameter at infinity, the relative velocity at infinity, the initial binary semimajor axis, and the critical binary velocity vc2 $=(3 / 2) \mathrm{Gm} / \mathrm{a}$, respectively. Black: Exchange interactions not leading to a merger. Blue: Interactions leading to a 3-body merger. Red: Interactions leading to a 2-body merger. Grey: Remaining set. The merger time-limit is set to $\mathrm{tGW}<$ tint $=105 \mathrm{yr}$. In our setup, the binary black hole and the incoming black hole are co-rotating and counter-rotating above and below the white dotted horizontal line, respectively. 

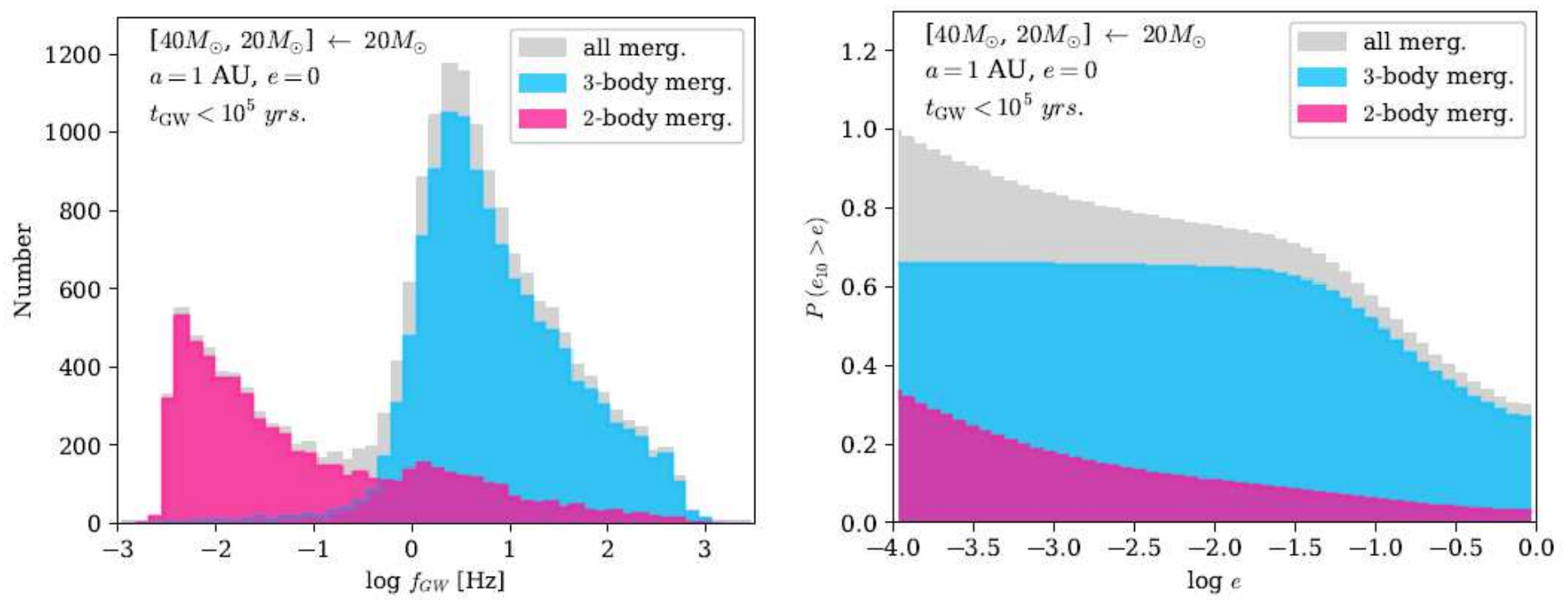

\section{Figure 4}

Eccentricity and gravitational wave frequency distributions. Results from 105 inde-pendent co-planar 2.5PN-scatterings, between an incoming $20 \mathrm{M}$ black hole and a [40M, 20M] binary black hole with an initial semi-major axis and eccentricity of $\mathrm{a}=1 \mathrm{AU}$ and $\mathrm{e}=0$, respec-tively. The red and blue distributions show the outcome from 2-body mergers and 3-body mergers, respectively, where grey is the total number. We only include binary black holes with resultant inspiral times of $\mathrm{tGW}<\mathrm{tint}=105 \mathrm{yr}$. Left: Distribution of binary black hole gravitational wave peak frequency, $\mathrm{fGW}$, measured at the time of assembly. Right: Cumulative distribution of binary black hole orbital eccentricities propagated to $10 \mathrm{~Hz}$. For sources formed with $\mathrm{fGW}>10 \mathrm{~Hz}$, the corresponding eccentricity has been set to $\mathrm{e}=1$. 